\title{
UM ESTUDO DE CASO SOBRE A PREVALÊNCIA DE TRANSTORNOS MENTAIS COMUNS EM ESTUDANTES DO ENSINO SUPERIOR
}

\section{ARTIGO ORIGINAL}

MENGALI, Priscila Macedo ${ }^{1}$, FERRAZ, Fernando Toledo ${ }^{2}$

MENGALI, Priscila Macedo. FERRAZ, Fernando Toledo. Um estudo de caso sobre a prevalência de transtornos mentais comuns em estudantes do ensino superior. Revista Científica Multidisciplinar Núcleo do Conhecimento. Ano 06, Ed. 07, Vol. 02, pp. 57-71. Julho de 2021. ISSN: 2448-0959, Link de acesso: https://www.nucleodoconhecimento.com.br/psicologia/mentais-comuns, $\quad$ DOI: 10.32749/nucleodoconhecimento.com.br/psicologia/mentais-comuns

\section{RESUMO}

No campus do Cefet/RJ Nova Friburgo foi observado um aumento de comportamentos característicos de sofrimento mental entre os estudantes do ensino superior. Tendo em vista essa observação, a presente pesquisa teve por objetivo verificar a prevalência de transtornos mentais comuns (TMC) entre esses estudantes. Foi utilizado o estudo de caso como estratégia de investigação, e a coleta de dados foi realizada a partir de um questionário sociodemográfico e acadêmico e o teste Self Report Questionnaire -SRQ-20. Na realização do tratamento estatístico foram utilizados o teste de independência qui-quadrado e a análise de regressão múltipla. A partir dos resultados pôde-se concluir que os estudantes do gênero feminino e os estudantes LGBTQI+ apresentaram uma maior prevalência de TMC. Na análise do teste qui-quadrado apresentaram maior prevalência de TMC: os estudantes solteiros, pardos, que residem em república e que estão no início dos seus cursos, entre o $1^{\circ} \mathrm{e}$

\footnotetext{
${ }^{1}$ Mestrado profissional em andamento em Sistemas de Gestão, Pós graduação lato sensu em Saúde Mental e Atenção Psicossocial e Graduação em Psicologia.

${ }^{2}$ Orientador. Doutorado em Engenharia de Produção.
} 
3o períodos. Na análise da regressão múltipla os dados mostraram que as variáveis Orientação Sexual, Gênero, Pressão externa que o estudante sofre e Expectativas quanto à inserção profissional foram estatisticamente significativas na suspeição de TMC.

Palavras-chave: estudantes do ensino superior, saúde mental, transtornos mentais comuns.

\section{INTRODUÇÃO}

A vida acadêmica pode envolver fatores de risco para a saúde mental dos indivíduos e com isto desencadear alterações emocionais, sofrimento psíquico e possíveis transtornos mentais tendo em vista a ocorrência de diversas mudanças relacionadas à rotina desses indivíduos.

As demandas e as exigências da vida universitária demonstram que o estudante, desde o seu ingresso na instituição de ensino, deve apresentar recursos cognitivos e emocionais complexos para manejar as demandas desse novo ambiente (PADOVANI et al., 2014).

A junção de vários fatores como situações estressantes, cobranças, a perda de referências quando o estudante tem que mudar de cidade e/ou Estado, a falta de sentido no decorrer dos estudos, as competições entre os próprios estudantes universitários podem favorecer o sofrimento psíquico.

O presente artigo surge diante da percepção da autora do aumento da ocorrência de comportamentos característicos de sofrimento mental entre os estudantes do ensino superior dentro do espaço físico do Cefet/RJ campus Nova Friburgo. O Cefet/RJ configura-se como instituição de ensino superior pluricurricular, especializada na oferta de educação tecnológica nos diferentes níveis e modalidades de ensino, caracterizando-se pela atuação prioritária na área tecnológica (PDI, 2015-2019). 
Considerando o aumento dessa demanda, esse trabalho teve como objetivo verificar a prevalência de transtornos mentais comuns entre os estudantes do ensino superior e analisar sua relação entre os fatores sociodemográficos e acadêmicos.

Conceito formulado por Goldberg e Huxley (1992), os transtornos mentais comuns são um conjunto de sintomas não psicóticos que caracterizam um sofrimento mental, porém não se encontram dentro dos critérios diagnósticos presentes nas classificações internacionais. Dentre esses sintomas podemos citar a irritabilidade, a insônia e a dificuldade de concentração. (MURCHO et al., 2016).

Os estudantes universitários são considerados como um grupo especial de investimento social do país, portanto é fundamental que se realizem estudos focados nessa área, dando ênfase às dimensões de maior vulnerabilidade nessa fase da vida com objetivo de identificar os fatores que predispõem a ocorrência do estresse emocional e, consequentemente, sua influência na saúde mental dos estudantes (PADOVANI et al., 2014).

O presente artigo está estruturado em quatro seções, a primeira apresenta alguns estudos sobre a prevalência dos transtornos mentais comuns em estudantres universitários. Na segunda seção, apresenta-se a metodologia de pesquisa adotada para este estudo, apresentando a classificação científica da pesquisa, a estratégia de investigação, o material utilizado, o universo e amostra, os instrumentos de coleta de dados e, por fim, a análise e o tratamento dos dados. Na terceira são apresentados os resultados e a discussão a partir da aplicação do questionário sociodemográfico e acadêmico e o teste Self Report Questionnaire -SRQ-20. Para o tratamento estatístico dos dados foram utilizados o teste de independência qui-quadrado e a análise de regressão múltipla. Na quarta e última seção são apresentadas as considerações finais do artigo alinhadas ao objetivo da pesquisa.

\section{ASPECTOS TEÓRICOS DO ESTUDO}

O Relatório da Organização Pan-Americana da Saúde da Região das Américas, informa que os transtornos mentais respondem por $34 \%$ das deficiências com pouca 
variação no nível nacional. Na América Latina e no Caribe, os problemas de saúde mental, incluindo o uso de substâncias psicoativas, respondem por mais de um terço da incapacidade total na Região. Desses, os transtornos depressivos estão entre as maiores causas de incapacidade, seguidos pelos transtornos de ansiedade (Pan American Health Organization - PAHO, 2018).

A Organização Mundial da Saúde (OMS), orientada pela preocupação com os impactos que os problemas de saúde mental poderiam apresentar em países periféricos, desenvolveu o Self-Reporting Questionnaire (SRQ-20) com o objetivo de avaliar os transtornos mentais comuns nesses países (SANTOS et al., 2009).

Os transtornos mentais comuns, conhecidos também como transtornos psiquiátricos menores, representam os quadros menos graves e mais frequentes de transtorno mental (TÓFOLI, 2006). Estão relacionados ao estresse, depressão e ansiedade, os quais pela sua elevada prevalência nos CSP (Cuidados de Saúde Primários) são considerados como um dos maiores problemas de saúde pública mundial (MURCHO et al., 2016).

Os transtornos mentais comuns são muito onerosos para a qualidade de vida dos indivíduos e para os seus relacionamentos interpessoais, além de ser potencial causa para o desenvolvimento de doenças mentais, como, por exemplo, transtornos somatoformes, ansiedade e depressão, podendo estar associados também a alterações do padrão do sono e a doenças crônicas (FERREIRA; KLUTH COVSKY; CORDEIRO, 2016).

Através da revisão integrativa da literatura científica nacional e internacional, Cerqueira e Graner (2019) traçaram um panorama sobre o sofrimento psíquico/transtornos mentais comuns (TMC) entre estudantes universitários. Os instrumentos mais utilizados para o rastreamento do sofrimento psíquico foram o Self Reporting Questionnarie (SRQ-20) e o General Health Questionnaire, que identificaram prevalências de $18,5 \%$ a $49,1 \%$. 
No Brasil, as prevalências de transtornos mentais comuns em universitários foram superiores ao identificado na população geral e entre os adolescentes, porém inferiores às obtidas em estudos com usuários da Atenção Primária à Saúde (CERQUEIRA; GRANER, 2019). Características da vida acadêmica e relacionais foram as que mais frequentemente associaram-se à presença de sofrimento psíquico entre universitários. Isso demonstra a importância do planejamento de intervenções que favoreçam o bem-estar dos estudantes e possibilitem vivências mais positivas no ambiente educacional (CERQUEIRA; GRANER, 2019).

O estudo de Padovani et al. (2014) buscaram identificar indicadores de vulnerabilidade e bem-estar psicológicos em estudantes universitários de vários cursos de graduação de seis instituições de ensino superior públicas e privadas de três Estados brasileiros. Os sinais e sintomas indicadores de estresse, burnout, ansiedade e depressão na população estudada se revelaram comuns. Identificou-se a prevalência de estresse de $52,88 \%$ entre os 783 alunos investigados; $13,5 \%$ dos estudantes apresentaram sintomas significativos de ansiedade; $7,26 \%$ dos estudantes apresentaram sintomas moderados a graves de depressão e aproximadamente $40 \%$ dos estudantes apresentaram sintomas de sofrimento psicológico. Diante disso e tendo em vista as implicações que o sofrimento psicológico pode acarretar a aprendizagem e formação do estudante universitário, é de fundamental importância que a universidade desenvolva ações integradas de prevenção e tratamento para esse público (PADOVANI et al., 2014).

Ferreira; Kluthcovsky e Cordeiro (2016) afirmaram que a prevalência de transtornos mentais comuns entre os estudantes de Medicina é alta e há uma escassez de estudos comparativos sobre esses transtornos.

\section{METODOLOGIA}

Trata-se de uma pesquisa de levantamento do tipo descritiva, que utilizou como estratégia de investigação o estudo de caso. No que diz respeito aos objetivos, a pesquisa caracteriza-se como de natureza exploratória, pois a finalidade é identificar o perfil sociodemográfico e acadêmico dos respondentes e, a partir daí, verificar quais 
dessas variáveis podem estar relacionadas a suspeição de transtornos mentais comuns nos estudantes.

Para a realização deste estudo, foi feita revisão de literatura para conferir um suporte teórico ao estudo. A revisão foi desenvolvida com base em artigos selecionados no Portal de Periódicos Capes, na base de dados Web of Science e Google Scholar além de busca em outras fontes: dissertações, teses, livros e outras fontes oficiais.

Para levantamento de dados dos alunos foi construído o questionário sociodemográfico e acadêmico de questões fechadas e os níveis de suspeição de transtornos mentais comuns foram levantados a partir do teste Self Report Questionnaire (SRQ-20). Foi considerado o número de 7 respostas "sim" como ponto de corte, conforme o estudo de Gonçalves; Stein e Kapczinski (2008).

O projeto de pesquisa foi submetido ao Comitê de Ética em Pesquisa da Universidade Federal Fluminense - Campus de Nova Friburgo, sendo aprovado sob o número de parecer: 4.326.970. Os procedimentos utilizados nesse estudo estão de acordo com a Resolução 466/2012 do Conselho Nacional de Saúde (CNS), que trata das diretrizes e normas regulamentadoras de pesquisas envolvendo seres humanos. Tão logo obtida essa aprovação foi realizado o pré-teste e em seguida a pesquisa foi enviada a todos os alunos ativos dos cursos de ensino superior, totalizando 754 alunos. $O$ teste e o questionário ficaram disponíveis para acesso dos respondentes durante 15 dias e obteve um total de 94 respondentes. A população da pesquisa foi composta pelos estudantes dos cursos de ensino superior do Cefet/RJ campus Nova Friburgo. Na análise e interpretação dos dados foi realizado o processamento de dados em planilha do Microsoft Excel e realizado tratamento estatístico com o teste de independência qui-quadrado e a análise de regressão múltipla.

\section{RESULTADOS E DISCUSSÃO}

Do total da amostra, 59,6 \% são do sexo masculino e a média de idade observada foi de 25 anos. Aproximadamente 72,34\% dos alunos estão na faixa etária entre 21 e 40 
anos, aproximadamente $22,34 \%$ entre 18 e 21 anos e os demais possuem mais de 40 anos.

O questionário foi respondido em sua maioria por alunos do curso de Bacharelado em Sistemas de Informação (37,2\%), seguido dos cursos de Tecnologia em Gestão de Turismo (24,5\%), Licenciatura em Física (21,3\%) e Engenharia Elétrica (17\%).

A tabela 1 apresenta a prevalência das queixas (perguntas) dentro dos grupos de sintomas psíquicos nos estudantes do ensino superior. Para realizar esse agrupamento de sintomas foi utilizado como referência o trabalho de Trajano (2018) e de Alves et al. (2015). Observa-se que no grupo de sintomas humor depressivo/ansioso a queixa sentir-se nervoso(a), tenso(a) ou preocupado(a) se destacou com $72,3 \%$ entre os estudantes pesquisados, o que também se verificou na pesquisa de Alves et al. (2015); Arantes (2018) e de Ferreira; Kluthcovsky e Cordeiro (2016). No grupo de sintomas somáticos predominou a queixa de cansar-se com facilidade (54,3\%). No grupo de sintomas de decréscimo de energia vital e no de pensamentos depressivos evidenciam-se, respectivamente, a queixa na dificuldade de tomar decisões $(58,5 \%)$ e a perda de interesse pelas coisas $(50 \%)$.

Tabela 1: Prevalência das queixas dentro dos grupos de sintomas psíquicos de acordo com o Self Reporting Questionnaire (SRQ-20)

\begin{tabular}{|l|l|l|}
\hline Grupos de sintomas & $\begin{array}{l}\text { Sim } \\
(\%)\end{array}$ & $\begin{array}{l}\text { Não } \\
(\%)\end{array}$ \\
\hline Humor depressivo/ansioso & & \\
\hline Q-4 Assusta-se com facilidade? & $33,0 \%$ & $67,0 \%$ \\
\hline Q-6 Sente-se nervoso (a), tenso (a) ou preocupado (a) & $72,3 \%$ & $27,7 \%$ \\
\hline Q-9 Tem se sentido triste ultimamente? & $57,4 \%$ & $42,6 \%$ \\
\hline Q-10 Tem chorado mais do que costume? & $21,3 \%$ & $78,7 \%$ \\
\hline Sintomas somáticos & & \\
\hline Q-1 Você tem dores de cabeça frequente? & $37,2 \%$ & $62,8 \%$ \\
\hline Q-2 Tem falta de apetite? & $20,2 \%$ & $79,8 \%$ \\
\hline Q-3 Dorme mal? & $47,9 \%$ & $52,1 \%$ \\
\hline
\end{tabular}




\begin{tabular}{|l|l|l|}
\hline Q-5 Tem tremores nas mãos? & $30,9 \%$ & $69,1 \%$ \\
\hline Q-7 Tem má digestão? & $25,5 \%$ & $74,5 \%$ \\
\hline Q-19 Você se cansa com facilidade? & $54,3 \%$ & $45,7 \%$ \\
\hline Decréscimo de energia vital & & \\
\hline Q-8 Tem dificuldades de pensar com clareza? & $34,0 \%$ & $66,0 \%$ \\
\hline $\begin{array}{l}\text { Q-11 Encontra dificuldades para realizar com satisfação suas } \\
\text { atividades diárias? }\end{array}$ & $56,4 \%$ & $43,6 \%$ \\
\hline Q-12 Tem dificuldades para tomar decisões? & $58,5 \%$ & $41,5 \%$ \\
\hline $\begin{array}{l}\text { Q-13 Tem dificuldades no serviço (seu trabalho é penoso, Ihe } \\
\text { causa- sofrimento?) }\end{array}$ & $12,8 \%$ & $87,2 \%$ \\
\hline Q-18 Sente-se cansado (a) o tempo todo? & $46,8 \%$ & $53,2 \%$ \\
\hline Q-20 Têm sensações desagradáveis no estomago? & $34,0 \%$ & $66,0 \%$ \\
\hline Pensamentos depressivos & & \\
\hline Q-14 É incapaz de desempenhar um papel útil em sua vida? & $29,8 \%$ & $70,2 \%$ \\
\hline Q-15 Tem perdido o interesse pelas coisas? & $50,0 \%$ & $50,0 \%$ \\
\hline Q-16 Você se sente uma pessoa inútil, sem préstimo? & $29,8 \%$ & $70,2 \%$ \\
\hline Q-17 Tem tido ideia de acabar com a vida? & $14,9 \%$ & $85,1 \%$ \\
\hline
\end{tabular}

Fonte: Elaborado pela própria autora (2020)

No teste qui-quadrado foi criada uma variável TMC (transtorno mental comum) a partir do escore total de cada respondente no SRQ-20, classificando-os como suspeitos de TMC ou não, de acordo com o ponto de corte selecionado: maior igual a 7. Foram consideradas estatisticamente significativas as associações entre as variáveis analisadas e a variável TMC cujo valor foi menor que o nível de significância estabelecido, neste caso, $p<0,05$ (tabela 2).

Em relação à saúde mental dos estudantes observa-se que a prevalência geral de TMC, de acordo com o ponto de corte adotado, foi de $50 \%$ observando-se uma diferença estatística significativa na presença de TMC em relação ao gênero: $71 \%$ das mulheres apresentaram TMC enquanto nos homens a prevalência foi de $35,7 \%$. Os transtornos mentais comuns vêm apresentando destaque, principalmente na população feminina. O sofrimento psíquico entre elas pode ser intensificado devido a alguns fatores do ciclo de vida e a dupla jornada trabalho associados aos 
componentes emocionais (PINHO; ARAÚJO, 2012). Em relação ao resultado do teste, observa-se a correlação entre gênero e TMC (.

Quanto à orientação sexual há uma predominância de respondentes heterossexuais, aproximadamente $84 \%$. Entre os LGBTQI+, $80 \%$ apresentaram suspeição de TMC, quase o dobro em relação aos heterossexuais. Em relação ao resultado do teste, observa-se a correlação entre orientação sexual e TMC (.

As variáveis cor, estado civil, curso, período e residência não apresentaram correlação, ou seja, não foram encontradas evidências de associação entre os transtornos mentais comuns e essas variáveis. Porém é importante destacar que um percentual elevado de estudantes (75\%) que moram em república, pensão, casa e/ou apartamento dividido apresentaram suspeição de transtornos mentais comuns.

Outro dado relevante está relacionado a variável cor: mais de $50 \%$ dos respondentes são brancos e apenas 1 respondente se declarou da cor amarela e nenhum da cor indígena. Dessa forma, o teste de independência qui-quadrado foi realizado considerando apenas as respostas correspondentes as cores/raças branca, parda e preta.

Tabela 2. Análise univariada entre as variáveis sociodemográficas e acadêmicas e os TMC

\begin{tabular}{|l|l|l|l|l|}
\hline Variáveis & $\mathrm{n}$ & $\%$ & $\%$ TMC & $p$ \\
\hline Gênero & 0,000771626 & & \\
\hline Feminino & 38 & 40,4 & 71 & \\
\hline Masculino & 56 & 59,6 & 35,7 & \\
\hline Orientação Sexual & 0,0112550486 & \\
\hline Heterossexual & 79 & 84 & 44 \\
\hline LGBTQ+ & 15 & 16 & 80 & \\
\hline Cor & 0,539205762 & \\
\hline Amarela & 1 & 1,1 & - & \\
\hline Branca & 50 & 53,2 & 46 & \\
\hline Indígena & 0 & 0 & 0 & \\
\hline
\end{tabular}




\begin{tabular}{|c|c|c|c|c|}
\hline Parda & 27 & 28,7 & 59 & \\
\hline Preta & 16 & 17 & 50 & \\
\hline Estado Civil & & & & 0,375074307 \\
\hline Solteiro & 79 & 84 & 50,6 & \\
\hline Casado/União estável & 11 & 11,7 & 36 & \\
\hline Divorciado & 0 & 0 & 0 & \\
\hline Outro & 4 & 4,3 & - & \\
\hline Curso & & & & 0,115793085 \\
\hline Engenharia Elétrica & 16 & 17 & 25 & \\
\hline Licenciatura em Física & 20 & 21,3 & 60 & \\
\hline Sistemas de Informação & 35 & 37,2 & 48,5 & \\
\hline Turismo & 23 & 24,5 & 60,8 & \\
\hline Período & & & & 0,402890322 \\
\hline Entre o $1^{\circ}$ e o $3^{\circ}$ período & 44 & 46,8 & 56,8 & \\
\hline Entre o 4 e o 6ํporíodo & 36 & 38,3 & 41,6 & \\
\hline Entre o $7^{\circ}$ e o $10^{\circ}$ período & 14 & 14,9 & 50 & \\
\hline Residência & & & & 0,626659865 \\
\hline Com pais & 62 & 65,9 & 46,77 & \\
\hline Outras pessoas da família & 7 & 7,5 & 57,14 & \\
\hline Cônjuge/companheiro & 12 & 12,8 & 50 & \\
\hline República, pensão & 8 & 8,5 & 75 & \\
\hline Sozinho & 5 & 5,3 & 40 & \\
\hline Outro & 0 & 0 & & \\
\hline Total & 94 & $100 \%$ & $50 \%$ & $\longrightarrow$ \\
\hline
\end{tabular}

Fonte: Elaborado pela própria autora (2020)

$\mathrm{Na}$ análise de regressão múltipla ocorreu a recodificação dos dados transformando as variáveis qualitativas em variáveis quantitativas conforme apresentado no quadro 6 .

Quadro 6. Recodificação das variáveis qualitativas

Variáveis qualitativas

Feminino
Variáveis quantitativas

0 


\begin{tabular}{|c|c|c|}
\hline \multicolumn{2}{|r|}{ Masculino } & 1 \\
\hline \multirow[t]{2}{*}{ Orientação } & LGBTQ+ & 0 \\
\hline & Heterossexual & 1 \\
\hline \multirow[b]{2}{*}{$\begin{array}{l}\text { Têm filhos? } \\
\text { Você exerce alguma } \\
\text { atividade remunerada } \\
\text { atualmente? }\end{array}$} & Não & 0 \\
\hline & Sim & 1 \\
\hline \multirow[t]{2}{*}{ Fuma cigarro (tabaco)? } & Nunca & 0 \\
\hline & Raramente & 1 \\
\hline Consome bebida alcoólica? & Ocasionalmente & 2 \\
\hline \multirow{2}{*}{$\begin{array}{l}\text { Faz uso de medicamentos } \\
\text { psicotrópicos/psicoativos? }\end{array}$} & Frequente & 3 \\
\hline & Muito frequente & 4 \\
\hline \multirow{3}{*}{$\begin{array}{l}\text { Eu me identifico com o meu } \\
\text { curso. }\end{array}$} & Discordo totalmente & 0 \\
\hline & Discordo & 1 \\
\hline & Não concordo/nem discordo & 2 \\
\hline \multirow{2}{*}{$\begin{array}{l}\text { O horário das aulas é bom } \\
\text { para mim. } \\
\text { Eu tenho amigos no CEFET. }\end{array}$} & Concordo & 3 \\
\hline & Concordo totalmente & 4 \\
\hline $\begin{array}{l}\text { Eu me cobro pelo bom } \\
\text { desempenho no curso. }\end{array}$ & & \\
\hline $\begin{array}{l}\text { Eu sofro pressão externa } \\
\text { para ter um bom } \\
\text { desempenho no curso. }\end{array}$ & & \\
\hline
\end{tabular}




\section{Eu me sinto sobrecarregado \\ ao buscar atender as \\ exigências do curso.}

\section{As minhas expectativas quanto à inserção profissional após o término do curso são positivas.}

Fonte: Elaborado pela própria autora (2020)

Os testes foram realizados através da ferramenta de análise de dados disponível no Excel. No software Excel utilizado, a significância global (Teste ) é apresentada como de significação e as significâncias individuais das variáveis independentes são apresentadas através do valor-p de cada (CASTRO, 2019). Nesta pesquisa, para ter os $95 \%$ de significância, considerou-se como critério .

A tabela 3 apresenta o resultado final obtido após as regressões múltiplas realizadas.

Tabela 3. Resultado final da análise de regressão múltipla

\begin{tabular}{|l|l|l|}
\hline Resultados de Regressão & & \\
\hline $\boldsymbol{R}$-quadrado & 0,387861741259301 \\
\hline $\boldsymbol{R}$-quadrado ajustado & 0,360349909405787 \\
\hline $\boldsymbol{F}$ & 14,0979976660389 \\
\hline $\boldsymbol{F}$ de significância & $5,974510^{-9}$ & \multicolumn{1}{|c|}{$\boldsymbol{p}$} \\
\hline Variáveis independentes & $\beta$ & $8,8810^{-9}$ \\
\hline Interseção & 12,27056967 & 0,016898305 \\
\hline $\mathbf{1}$ - Gênero & $-2,40824766$ & 0,038482663 \\
\hline 2 - Orientação sexual & $-2,541245656$ & 0,010948766 \\
\hline 3 - Eu sofro pressão externa & 0,937472365 & 0,006528036 \\
\hline 4 - Expectativas quanto à inserção profissional & $-1,270550576$ & \\
\hline
\end{tabular}

Fonte: Elaborado pela própria autora (2020) 
Os resultados sugerem que as variáveis gênero, orientação sexual, pressão externa e expectativas quanto à inserção profissional estão relacionadas à suspeição de transtornos mentais comuns nos estudantes dos cursos analisados.

No tocante a variável gênero, observa-se uma correlação negativa com a variável dependente (sinal do coeficiente negativo), ou seja, quanto maior for o valor da variável gênero, menor será o valor de . Assim, o resultado indicado na regressão foi de que há maior suspeição de TMC em estudantes mulheres, o que está de acordo com os dados apresentados na tabela 3 e também em outras pesquisas, como as apresentadas por Jansen et. al. (2011); Alves et al. (2015) e Cunha (2014). Na pesquisa de Tran et al. (2017) com estudantes universitários da Universidade de Nice Sophia-Antipolis (UNSA) as estudantes do sexo feminino corriam maior risco de apresentar sintomas de ansiedade. Conforme Senicato, Azevedo e Barros (2018) além das diferenças biológicas e sociais, as mulheres experienciam desigualdades de gênero que tem consequências como o excesso de trabalho doméstico e aumento da violência contra a mulher.

Com relação à orientação sexual, observa-se novamente uma correlação negativa, o que indica uma maior suspeição de TMC em estudantes de orientação LGBTQI+. No estudo de Woodford, Kulick e Atteberry (2015) foram apresentadas as possíveis causas de sofrimento mental em estudantes LGBTQ+. Um dos resultados sugerem que o aumento da frequência de assédio heterossexista está associado a maior depressão e sintomas de ansiedade, além de risco de abuso de álcool nesses estudantes.

Outro resultado apresentado na regressão foi: quanto maior for a pressão externa para que o estudante tenha um bom desempenho no curso, maior será a sua tendência ao desenvolvimento de transtornos mentais comuns. Há uma correlação positiva entre a variável pressão externa e a variável dependente. No estudo com universitários brasileiros, Ariño e Bardagi (2018) concluíram que há relações estatisticamente significativas entre níveis elevados de stress, ansiedade e depressão com a qualidade das vivências acadêmicas e a autoeficácia para formação superior, ou seja, a sobrecarga de demandas acadêmicas, assim como a percepção do estudante sobre 
sua capacidade de lidar com essas demandas, se constituem como um fator de risco para o adoecimento.

Observa-se ainda uma correlação indireta em relação às expectativas quanto à inserção profissional após o término do curso. Ou seja, quanto mais positivas forem as expectativas dos estudantes em relação a sua inserção profissional, menor será o indicativo de suspeição de TMC. O estudo de Costa et al. (2014) envolvendo universitários do último ano de 3 cursos da área de saúde mostrou um percentual aumentado de TMC (33,7\%). O que difere da pesquisa de Alfonso et al. (2013) que apresenta entre as dificuldades dos estudantes os altos níveis de estresse devido a insatisfação e frustração decorrente das altas expectativas acadêmicas no início dos cursos e não no final.

\section{CONSIDERAÇÕES FINAIS}

Os resultados apresentados nesse estudo mostraram que há correlação entre variáveis sociodemográficas e acadêmicas e a prevalência de transtornos mentais comuns em estudantes do ensino superior.

No agrupamento de sintomas realizados a partir da análise do SRQ-20 foi observado a predominância das seguintes queixas entre os estudantes: "sente-se nervoso, tenso ou preocupado" (72,3\%), "tem dificuldades para tomar decisões" (58,5\%), "você se cansa com facilidade" (54,3\%) e "tem perdido interesse pelas coisas" (50\%).

A partir das duas análises estatísticas utilizadas nessa pesquisa, foi possível concluir a prevalência de TMC em estudantes do gênero feminino e em estudantes LGBTQI+. A utilização do teste qui-quadrado na análise estatística univariada mostrou um maior percentual de suspeição de TMC em estudantes pardos, solteiros e que residem em república e que estão iniciando seus cursos, entre o primeiro e terceiro períodos.

$\mathrm{Na}$ análise de estatística multivariada (regressão múltipla) os dados sugerem que as variáveis Gênero, Orientação Sexual, Pressão externa que o estudante sofre e Expectativas quanto à inserção profissional mostram-se estatisticamente significativas 
na suspeição de TMC. Nessa análise foi possível observar também que quanto mais o estudante sofre pressão externa, maior é a prevalência de TMC e que quanto mais positivas forem as expectativas dos alunos em relação a sua inserção profissional, menor será o indicativo de suspeição de TMC.

Os resultados dessa pesquisa podem subsidiar intervenções que permitam oferecer uma maior proteção e apoio aos grupos que apresentaram maior percentual de suspeição de TMC, sendo considerados os grupos de maior risco dentro do Cefet/RJ campus Nova Friburgo.

Em relação às limitações da pesquisa, pode-se considerar o tamanho da amostra. Num universo de 754 estudantes de ensino superior, apenas 12,46\% responderam a pesquisa, um percentual abaixo do esperado. Esse percentual talvez se justifique tendo em vista o contexto da pandemia do COVID-19, que além de ter trazido mudanças para vida de todos, também impossibilitou um contato mais direto da pesquisadora com os estudantes.

Outras limitações que podem ser consideradas são os motivos pelos quais determinados grupos foram identificados com maior percentual de suspeição de TMC. Porém, os resultados dessa pesquisa tornam-se impulsionadores para que pesquisas futuras explorem essa temática de forma mais profunda.

\section{REFERÊNCIAS}

ALFONSO, S. et. al. Perfiles de expectativas académicas en alumnos españoles y portugueses de enseñanza superior. Revista Galego-portuguesa de Psicoloxía e Educación, v. 21, n. 1, p. 125-136, 2013.

ALVES, A. P. et. al. Prevalência de transtornos mentais comuns entre profissionais de saúde. Revista Enfermagem UERJ, Rio de Janeiro, v. 23, n. 1, p. 64-69, 2015.

\section{ARANTES, A. P. Qualidade de vida e transtorno mental comum em estudantes} graduandos de ciências da saúde. 2018. Dissertação (Mestrado) - Mestrado em 
Atenção à Saúde, Universidade Federal do Triângulo Mineiro, Uberaba, 2018. Disponível em: http://bdtd.uftm.edu.br/handle/tede/745. Acesso em: 20 nov. 2020.

ARIÑO, D. O.; BARDAGI, M. P. Relação entre fatores acadêmicos e a saúde mental de estudantes universitários. Revista Psicologia em Pesquisa, Juiz de Fora, v.12, n. 3, p. 44-52, 2018.

CENTRO FEDERAL DE EDUCAÇÃO TECNOLÓGICA CELSO SUCKOW DA FONSECA. Plano de Desenvolvimento Institucional - PDI 2015-2019. Rio de Janeiro. http://www.cefetrj.br/attachments/article/97/PDI\%2020152019_versa\%CC\%830\%20fi nal\%20revisada\%20(2).pdf Acesso em: 31 jan. 2020.

CERQUEIRA, A. T. de A. R.; GRANER, K. M. Revisão integrativa: sofrimento psíquico em estudantes universitários e fatores associados. Ciência \& Saúde Coletiva, v. 24, n.4, p.1327-1346, 2019.

COSTA, E. F. de O. et al. Common mental disorders and associated factors among final-year healthcare students. Revista da Associação Médica Brasileira, v. 60, n.6, p. 525-530, 2014.

CUNHA, M. A. de S. Aplicação do SRQ20 e protocolo de avaliação psicológica em pacientes com hanseníase. 2014. Dissertação (Mestrado) - Mestrado em Ciências da Saúde, Universidade Federal de Uberlândia, Uberlândia, 2014. Disponível

em: https://repositorio.ufu.br/bitstream/123456789/12809/1/AplicacaoSRQ20Protocolo.pd fAcesso em: 02 nov. 2019.

FERREIRA, C. M. G.; KLUTHCOVSKY, A. C. G. C.; CORDEIRO, T. M. G. Prevalência de Transtornos Mentais Comuns e Fatores Associados em Estudantes de Medicina: um Estudo Comparativo: Revista Brasileira de Educação Médica, v.40, n.2, p. 268277, 2016. 
GONÇALVES, D.M.; STEIN, A.T.; KAPCZINSKI, F. Avaliação de desempenho do Self-Reporting Questionnaire como instrumento de rastreamento psiquiátrico: um estudo comparativo com o Structured Clinical Interview for DSM-IV-TR. Cadernos de Saúde Pública. v. 24, n. 2, p. 380-390, 2008.

JANSEN, K. et. al. Transtornos mentais comuns e qualidade de vida em jovens: uma amostra populacional de Pelotas. Caderno de Saúde Pública, v.27 , p. 440-448, 2011.

MURCHO, N.; PACHECO, E.; JESUS, S. N. de. Transtornos mentais comuns nos Cuidados de Saúde Primários: Um estudo de revisão. Revista portuguesa de enfermagem de saúde mental, Porto, n. 15, p. 30-36, 2016.

PADOVANI, R. C. et. al. Vulnerabilidade e bem-estar psicológicos do estudante universitário. Revista Brasileira de Terapias Cognitivas, v. 10, n.1, p. 02-10, 2014.

PAN AMERICAN HEALTH ORGANIZATION. The Burden of Mental Disorders in the Region of the Americas, 2018. Washington, D.C.: PAHO; 2018. Disponível em: https://www.paho.org/bra/index.php?option=com_content. Acesso em: 27 set. 2019.

PINHO, P. de S.; ARAÚJO, T. M. de. Associação entre sobrecarga doméstica e transtornos mentais comuns em mulheres. Revista Brasileira de Epidemiologia, v.15, n.3, p. 560-572, set. 2012.

SANTOS, K. O. B.; ARAÚJO, T. M. de A.; OLIVEIRA, N. F. de. Estrutura fatorial e consistência interna do Self-Reporting Questionnaire (SRQ-20) em população urbana.

Cadernos de Saúde Pública, Rio de Janeiro, v. 25, n. 1, p. 214-222, 2009.

SENICATO, C.; AZEVEDO, R. C. S.; BARROS, M. B. A. Transtorno mental comum em mulheres adultas: identificando os segmentos mais vulneráveis. Ciência Saúde Coletiva, v.23, n.8, p.2543-2554, 2018. 
TÓFOLI, L. F. F. Transtornos somatoformes, síndromes funcionais e sintomas físicos sem explicação. In: LOPES, A.C., organizador. Tratado de clínica médica. São Paulo: Roca; 2006.

TRAJANO, S. da S., Aspectos psicossociais, consumo de drogas e transtorno mental comum em ambiente universitário. 2018. Dissertação (Mestrado) Programa de PósGraduação em Saúde Coletiva, Universidade de Fortaleza, Fortaleza, 2018.2 Disponível em: https:/uol.unifor.br/oul/conteudosite/F10663420190218130739106518/Dissertacao.p df Acesso em: 18 fev. 2020.

TRAN, A. et al. Health assessment of French university students and risk factors associated with mental health disorders. PLOS ONE. v. 12, n. 11, p. 1-18, 2017.

WOODFORD, M. R., KULICK, A., ATTEBERRY, B. Protective Factors, Campus Climate, and Health Outcomes Among Sexual Minority College Students. Journal of Diversity in Higher Education. v. 8, n. 2, p. 73-87, 2015.

Enviado: Maio, 2021.

Aprovado: Julho, 2021. 